Article

\title{
A New Proof of a Conjecture on Nonpositive Ricci Curved Compact Kähler-Einstein Surfaces
}

\author{
Zhuang-Dan Daniel Guan \\ Department of Mathematics, The University of California at Riverside, Riverside, CA 92521, USA; \\ zguan_02@yahoo.com
}

Received: 26 December 2017; Accepted: 26 January 2018; Published: 7 February 2018

\begin{abstract}
In an earlier paper, we gave a proof of the conjecture of the pinching of the bisectional curvature mentioned in those two papers of Hong et al. of 1988 and 2011. Moreover, we proved that any compact Kähler-Einstein surface $M$ is a quotient of the complex two-dimensional unit ball or the complex two-dimensional plane if (1) $M$ has a nonpositive Einstein constant, and (2) at each point, the average holomorphic sectional curvature is closer to the minimal than to the maximal. Following Siu and Yang, we used a minimal holomorphic sectional curvature direction argument, which made it easier for the experts in this direction to understand our proof. On this note, we use a maximal holomorphic sectional curvature direction argument, which is shorter and easier for the readers who are new in this direction.
\end{abstract}

Keywords: Kähler-Einstein metrics; compact complex surfaces; pinching of the curvatures

\section{Introduction}

In [1], the authors conjectured that any compact Kähler-Einstein surface with negative bisectional curvature is a quotient of the complex two-dimensional unit ball. They proved that there is a number $a \in(1 / 3,2 / 3)$ such that if at every point $P, K_{a v}-K_{\min } \leq a\left(K_{\max }-K_{\min }\right)$, then $M$ is a quotient of the complex ball. Here, $K_{\min }\left(K_{\max }, K_{a v}\right)$ is the minimal (maximal, average) of the holomorphic sectional curvature. The number $a$ they obtained is $a<\frac{2}{3[1+\sqrt{6 / 11}]}$ (almost 0.38; see [2], p. 398). In [3], Yi Hong pointed out that this is also true if $a \leq \frac{2}{3[1+\sqrt{1 / 6}]}<0.476$. (For this part, this is due to Professor Hong. One note that he was the first author there.) We also observed in Theorem 2 that if $a \leq \frac{1}{2}$, then there is a ball-like point $P$. That is, at $P, K_{\max }=K_{\min }$. We notice that $\sqrt{1 / 6}>1 / 3$. Therefore, we conjectured in [3] that $M$ is a quotient of the complex ball if $a=\frac{1}{2}$. In general, we believe that we might not obtain a quotient of the complex ball if $a>\frac{1}{2}$. In [2,4], the author used a different method and proved that $a$ can be $\left(3+\frac{4 \sqrt{3}}{3}\right) / 11$ (almost 0.48 according to [5], p. 2628, just before Theorem 1.2); see [4] (p. 669) or [2] (p. 398). In [5], the authors improved the constant to $a<\frac{1}{2}$, which gave a proof of a weaker version of the conjecture.

In [6], we proved the following:

Proposition 1. Let $M$ be a connected compact Kähler-Einstein surface with nonpositive scalar curvature; if we have

$$
K_{a v}-K_{\min } \leq \frac{1}{2}\left[K_{\max }-K_{\min }\right]
$$

at every point, then $M$ is a compact quotient of either the complex two-dimensional unit ball or the complex two-dimensional complex plane.

For important mathematics work, it is common practice to give different and (possibly) simpler proofs (for certain experts and readers). For examples, see [7-14] and so forth. 
This note is for the experts who are new in this direction. In the second section, we review the basic material from [1] with an emphasis on the maximal holomorphic sectional direction instead of the minimal holomorphic sectional direction in $[1,3,5,6]$. We prove the existence of the ball-like points, as we did in the second section in [6], by using a different but similar function. In the third section, we again use Hong-Cang Yang's function and a different but similar calculation with respect to the maximal direction instead of the minimal direction. We give some detailed calculations in the Appendix as the last section of this paper.

\section{Existence of Ball-Like Points}

Here, we repeat the argument in the proof of our Proposition 1 given in [6] by using a different but similar argument:

Proposition 1 (cf. [3] pp. 597-599; [6] Proposition 1) Suppose that

$$
K_{a v}-K_{\min } \leq \frac{1}{2}\left[K_{\max }-K_{\min }\right]
$$

for every point on the compact Kähler-Einstein surface with nonpositive Ricci curvatures. There is at least one ball-like point.

Proof of Proposition 1. Throughout this section, as in $[1,5,6]$, we assume that $\left\{e_{1}, e_{2}\right\}$ is a unitary basis at a given point $P$ with

$$
\begin{gathered}
R_{1 \overline{1} 1 \overline{1}}=R_{2 \overline{2} 2 \overline{2}}=K_{\text {min }}, R_{1 \overline{1} 1 \overline{2}}=R_{2 \overline{2} 2 \overline{1}}=0 \\
A=2 R_{1 \overline{1} \overline{2} \overline{2}}-R_{1 \overline{1} 1 \overline{1}} \geq 0, B=\left|R_{1 \overline{2} 1 \overline{2}}\right|
\end{gathered}
$$

As in [1], we always have that $A \geq|B|$, and we assume that $B \geq 0$. This also implies, if the sectional curvatures have a $1 / 4$ pinching, that is, the section curvature is inside an interval $\left[-\frac{1}{4} a(P),-a(P)\right]$ at every point $P$ for a nonnegative function $a(P)$, that $M$ is covered by a ball. This was pointed out in [5]. This is because if we let $a(P)=-R_{1 \overline{1} 1 \overline{1}}, e_{i}=X_{i}+\sqrt{-1} Y_{i}$, then at least one of $R\left(X_{1}, X_{2}, X_{1}, X_{2}\right)$ and $R\left(X_{1}, Y_{2}, X_{1}, Y_{2}\right)$ is greater than or equal to $-\frac{1}{4} a(P)$. The same argument works for the higher-dimensional case. Our proposition is a kind of the generalization of the $1 / 4$ pinching.

If $P$ is not a ball-like point, according to [1], we can do the above for a neighborhood $U(P)$ of $P$ whenever $A>B$ (case 1 in [1], p. 475). In [6], we took great effort in handling the case in which $A=B$. We write

$$
\alpha=e_{1}=\sum a_{i} \partial_{i}, \beta=e_{2}=\sum b_{i} \partial_{i}
$$

and

$$
S_{1 \overline{1} 1 \overline{1}}=R\left(e_{1}, \bar{e}_{1}, e_{1}, \bar{e}_{1}\right)=\sum R_{i \bar{j} k \bar{l}} a_{i} \bar{a}_{j} a_{k} \bar{a}_{l}
$$

and so on. In particular, we have

$$
S_{1 \overline{1} 1 \overline{1}}=S_{2 \overline{2} 2 \overline{2}}=K_{m i n}, S_{1 \overline{1} 1 \overline{2}}=S_{2 \overline{2} 2 \overline{1}}=0
$$

According to [1], we have

$$
\begin{gathered}
K_{\text {max }}=K_{\text {min }}+\frac{1}{2}(A+B), K_{a v}=K_{\text {min }}+\frac{1}{3} A \\
\frac{1}{3}\left[K_{\text {max }}-K_{\text {min }}\right] \leq K_{a v}-K_{\text {min }} \leq \frac{2}{3}\left[K_{\text {max }}-K_{\text {min }}\right]
\end{gathered}
$$

This also shows that $A$ and $B$ are independent of the choice of $e_{1}$ and $e_{2}$. Additionally, our condition in Proposition 1 is therefore the same as $A \leq 3 B$.

In this section, we denote the maximal direction by $e_{1 *}$ and use ${ }^{*}$ in the notation of the corresponding terms' minimal direction case. We assume that $P$ is not a ball-like point. Under our 
assumption, $B>0$. According to ([1], p. 474), $e_{1^{*}}$ could be $\frac{1}{\sqrt{2}}\left(e_{1}+e_{2}\right)$. We could pick up $e_{2}=\frac{i}{\sqrt{2}}\left(e_{1}-e_{2}\right)$. We have

$$
\begin{gathered}
A^{*}=2 R_{1^{*} 1^{*} 2^{*} 2^{*}}-R_{1^{*} 1^{*} 1^{*} 1^{*}}=-\frac{1}{2}(A+3 B) \\
B^{*}=R_{1 * 2 * 1^{*} 2^{*}}=\frac{1}{2}(A-B)
\end{gathered}
$$

In our case, we have $A^{*}+3 B^{*}=A-3 B \leq 0$, that is, $-A^{*} \geq 3 B^{*}$. Moreover, from both the arguments in [1] (pp. 474-475), the choices of the directions of $e_{1^{*}}$ are isolated on the projective holomorphic tangent space. These two cases are case $1: A>B$; and case $2: A=B$. In case 1 , there is only one direction for the minimal holomorphic sectional curvature, and there is only one direction for the maximal holomorphic sectional curvature, because, by our assumption, $A \leq 3 B$; that is, $B$ is not zero at a nonball-like point. The second statement also follows from the argument in [1] by applying it to the maximal direction instead of the minimal direction. In case 2, there is a circle for the minimal direction, but there is a unique maximal direction. That is, one could always have a smooth frame of $e_{1^{*}}$. This might make the proof simpler. However, near the points with $B^{*}=A-B=0$, we might still have difficulty to obtain a smooth frame nearby such that $B^{*} \geq 0$. Therefore, we only assume that $B^{*} \geq 0$ at $P$, but this is not necessary true nearby if $B^{*}(P)=0$.

In $[3,6]$, we let $\Phi_{1}=\frac{|B|^{2}}{A^{2}}=\tau^{2}$. Here, we let $\Phi_{1}^{*}=\frac{\left|B^{*}\right|^{2}}{\left(A^{*}\right)^{2}}=\left(\tau^{*}\right)^{2}$ and $\tau^{*}=-\frac{\left|B^{*}\right|}{A^{*}} \geq 0$.

Our condition is the same as $\tau^{*} \leq 1 / 3$. If there is no ball-like point, there is a maximal point.

Now, $\tau^{*}=\frac{A-B}{A+3 B}=\frac{1}{3}\left(-1+\frac{4}{1+3 \tau}\right)$. The maximal of $\tau^{*}$ is just the minimal of $\tau$.

The calculation of the Laplace of $\Phi_{1}$ at a minimal point, which is not a ball-like point, and $A \neq B$ in [6] showed that $B^{*}=0$.

A similar calculation of the Laplacian of $\Phi_{1}^{*}$ with $B^{*} \neq 0$ shows that

$$
\Delta \Phi_{1}^{*}=6 A^{*}\left(\tau^{*}\right)^{2}\left(\left(\tau^{*}\right)^{2}-1\right)+h^{*}
$$

Here $\Delta \Phi_{1}^{*}$ has two general terms, just as for the formula for $\Delta \Phi_{1}$ in [6]; see the Appendix at the end of this paper. The first term is always nonnegative, as $\tau^{*} \leq \frac{1}{3} \leq 1$. The second term is a Hermitian form $h^{*}$ to $y^{*}$. We can separate $y^{*}$ into two groups: $y_{2 j}^{*}$ in one group and $y_{1 j}^{*}$ in the other. These two groups of variables are orthogonal to each other with respect to this Hermitian form. That is, $h^{*}=h_{1}^{*}+h_{2}^{*}$ with $h_{1}^{*}$ (or $h_{2}^{*}$ ) only depends on the first (second) group of variables.

We need to check the nonnegativity for each of these.

For $y_{11}^{*}, y_{12}^{*}$, the corresponding matrix of $h_{2}^{*}$ is

$$
\left[\begin{array}{cc}
2\left(9\left(\tau^{*}\right)^{2}-1\right)\left(\left(\tau^{*}\right)^{2}-1\right) & 0 \\
0 & 0
\end{array}\right]
$$

and the matrix for $h_{1}^{*}$ of $y_{21}^{*}, y_{22}^{*}$ is

$$
\left[\begin{array}{cc}
0 & 0 \\
0 & 2\left(9\left(\tau^{*}\right)^{2}-1\right)\left(\left(\tau^{*}\right)^{2}-1\right)
\end{array}\right]
$$

When $P$ is a critical point of $\Phi_{1}^{*}$, the matrices on $y^{*}$ are clearly semipositive. Therefore, if there is no ball-like point, then we have that at the maximal point of $\Phi_{1}^{*}, \tau^{*}=0$ or $A^{*}=0$, because $\tau^{*} \leq \frac{1}{3}$.

If $A^{*}=0$, then we have a ball-like point. Thus we are done.

On the other hand, if $\tau^{*}=0$, we have $B^{*}=0$ at $P$. Because $P$ is a maximal point for $\tau^{*}$, this implies that $B^{*}=0$ on the whole manifold. In this case, we could always assume that $B^{*} \geq 0$.

According to [1] (p. 475, case 2), that is, when $A=B$, we have smooth coordinates with $K_{\max }=R_{1 \overline{1} 1 \overline{1}}$ (this fortunately always works when $A=B$. In general, the original argument might 
not always work, as one might not have $A=B$ always nearby. However, as [1], case 1 also works for the maximal direction instead of the minimal direction, this implies that under our condition, the directions for $K_{\max }$ are always isolated. Therefore, it might be better for one to choose $K_{\max }$ instead of $K_{\text {min }}$ from the very beginning). Using this new coordinate, we can define the similar functions $A^{*}$ and $B^{*}$. In general, $B^{*}=\frac{1}{2}(A-B)$ and $A^{*}=-\frac{1}{2}(A+3 B)$. In our case, $B^{*}=0$ and $A^{*}=-2 A$. Using this new coordinate, one can do the calculation for any of the functions in [1,2,4] (or [5]; see the next section), for which the set of ball-like points is the whole manifold. If one does not like Polombo's function $\Phi_{\alpha}$ ([2], p. 418) with $\alpha=-\frac{8}{7}$ (e.g., [2], p. 417, Lemma), then one might simply use the function with $\alpha=-1$ (in $[2,4]$; not the vector we mentioned in this paper earlier), that is, the new function is proportional to $\Phi_{2}=(3 B-A) A$. In our case, this is just $2 A^{2}$. We can apply $\Phi_{2}^{\frac{1}{3}}$. This is relatively easy; thus we leave it to the readers (or see Equation (4) in the generalization). Actually, this paragraph is not needed for the proofs of Corollary 1 and Lemma 1. Additionally, in this special case, the original frame in [1] works. Thus, one could simply apply [1].

One can also use the function in [1] (p. 477):

$$
3 \gamma_{2}-\gamma_{1}^{2}=\frac{1}{2}\left(A^{2}+3 B^{2}\right)
$$

We can also still use the argument in [1], case 1, in which the minimal vectors are no longer isolated but are points in a smooth circle bundle over the manifold, such that we could choose a smooth section instead.

This paragraph is also not needed in the following Corollary 1 and Lemma 1 , as in these two propositions, we already have $A=3 B$. With $A=B$, one can readily obtain that $A=B=0$.

If $A=0, K_{\max }=K_{\min }$ and $P$ is a ball-like point, we have a contradiction. Therefore, the set of ball-like points is not empty.

$$
\text { Q. E. D. }
$$

We observe that if $A=3 B$ at $P$, then $\Phi_{1}$ achieves the minimal value at $P$ and $A \neq B$ unless $P$ is a ball-like point. That is, the first part of the proof of Proposition 1 goes through; thus, $P$ must be a ball-like point.

Corollary 1. Assuming the above, if $K_{a v}-K_{\min }=\frac{1}{2}\left(K_{\max }-K_{\min }\right)$ at $P$, then $P$ is a ball-like point.

Therefore, we have the following:

Lemma 1. If $K_{a v}-K_{\min } \leq \frac{1}{2}\left[K_{\max }-K_{\min }\right]$ on $M$, then we have $K_{a v}-K_{\min }<\frac{1}{2}\left[K_{\max }-K_{\min }\right]$ on $M-N$, where $N$ is the subset of all the ball-like points.

Therefore, we can apply the argument of [5]. To do this, one needs the following Proposition 4 in [1]:

Proposition 2. (cf. [1,3], Theorem 3.) If $N \neq M$, then $N$ is a real analytic subvariety and codim $N \geq 2$.

As in [1], Proposition 2 gives us a way to the conjecture by finding a superharmonic function on $M$, which was obtained by Hong-Cang Yang around 1992. In [1,3], the authors used $\Phi=6 B^{2}-A^{2}$. In [2], Polombo used $(11 A-3 B)(B-A)+16 A B$; see [2] (p. 417, Lemma. One might ask why we need another function but do not use our $\Phi_{1}$; the answer is that by a power of $\Phi_{1}$, we can only correct the Laplace by $\left|\nabla \Phi_{1}\right|^{2}$. However, this could only change the upper left coefficients of our matrices as it only provides $|x|^{2}$ terms. In the case of $\Phi_{1}$, it does not work, as $\frac{\tau}{A} \neq 0$ but the coefficients of $\left|y_{12}\right|^{2},\left|y_{21}\right|^{2}$ are zeros.

Therefore, we need another function, which was provided by Hong-Cang Yang. 
Remark 1. Whenever there is a bounded continuous nonnegative function $f$ on $M$ such that $(1) f(N)=0,(2)$ $f$ is real analytic on $M-N$, and (3) $\Delta f \leq 0$ on $M-N$, then $f=0$. Here $N$ could be just a codimension 2 subset. This is true in general for extending continuous superharmonic functions over a codimension 2 subset; see $[1,3,5]$. Here, we wish to give our own reasons as to why this is true in these special cases. If we define $M_{s}=\left\{\left.x \in M\right|_{\operatorname{dist}(x, N) \geq s}\right\}$ and $h_{s}=\partial M_{s}$, then the measure of $h_{s}$ is smaller than $O(s)$ when s tends to zero. Therefore,

$$
0 \geq \ln 2 \int_{M_{2 \delta}} \Delta f \omega^{n} \geq \int_{\delta}^{2 \delta}\left[\int_{M_{s}} \Delta f \omega^{n}\right] s^{-1} d s=\int_{\delta}^{2 \delta}\left[\int_{h_{s}} \frac{\partial f}{\partial n} d \tau\right] s^{-1} d s
$$

However, by applying an integration by parts to the single-variable integral, the last term is about $(\delta)^{-1} \int_{h_{2 \delta}}(f-g) d \tau \rightarrow 0$, as $f$ is bounded and $f-g$ tends to 0 near $N$, where $g$ is the $f$ value of the corresponding point on $h_{\delta}$. For example, if $f=r^{a}$ with $a>0$, then

$$
\frac{\partial f}{\partial n}=a r^{a-1}=a s^{a-1}
$$

and

$$
\int_{h_{s}} \frac{\partial f}{\partial n} d \tau=O\left(s^{a}\right) \rightarrow 0
$$

Therefore, $\Delta f=0$ on $M-N$. Therefore $f$ extends over $N$ as a harmonic function. This implies that $f=0$ on $M$.

Now, letting $f=(3 B-A)^{a}$, which is natural after the proof of Proposition 1 , we show in the next section that $\Delta f \leq 0$ for $a \leq \frac{1}{3}$ (see also a proof in [5]). Therefore, $A=3 B$ always. By Corollary 1 , we have $A=B=0$. This function is also related to the functions in [2] (p. 417) with $a_{1}=a_{3}=0$. In [2], Polombo had to pick up functions with $a_{1}=a_{2}$ to avoid a complication of the singularities; see [2] (p 406 and the first paragraph on p. 418); see also [4] (the last paragraph of p. 668). However, we completely resolve the difficulty in the next section.

\section{Generalized Hong-Cang Yang's Function}

We let $\Psi=3 B-A=-A^{*}-3 B^{*}$. Around 1992, Hong-Cang Yang considered $f=\Psi^{\frac{1}{3}}$. In [5], the authors had a formula for the Laplacian of $\Psi$. To apply the method to the maximal direction, we notice that the same formula holds. Moreover, if we let $\Psi_{k}=3 B+k A$, then $\Psi=\Psi_{-1}$ and we have the following:

Lemma 2. (cf. [5], p. 2630, Equation (13).) We have $A=\frac{3 B^{*}-A^{*}}{2}$,

$$
B=-\frac{A^{*}+B^{*}}{2}
$$

and

$$
\begin{aligned}
& \Delta(3 B+k A)=3\left[\Psi_{k} R_{1 \overline{1} 2 \overline{2}}-B(3 A+k B)\right] \\
& \quad+\frac{3}{B}\left|\nabla\left(\operatorname{Im} R_{1 \overline{2} 1 \overline{2}}\right)\right|^{2}+6\left[(B+k A) \sum|y|^{2}+2(A+k B) \operatorname{Re} \sum y_{i 1} \bar{y}_{i 2}\right]
\end{aligned}
$$

In particular, we have

$$
\begin{aligned}
\Delta \Psi & =3\left[\Psi R_{1 * 1^{*} 2^{*} 2^{*}}+B^{*}\left(3 A^{*}+B^{*}\right)\right] \\
& -\frac{3}{B^{*}}\left|\nabla\left(\operatorname{Im} R_{1^{*} 2^{*} 1^{*} 2^{*}}\right)\right|^{2}-6\left(A^{*}+B^{*}\right) \sum\left|y_{i 1}^{*}+y_{i 2}^{*}\right|^{2}
\end{aligned}
$$

It is clear that in the case of the maximal direction, we have to assume $B^{*} \neq 0$. That is, we still need to deal with the case in which $A=B$. This is because, in general, one cannot calculate the second 
derivatives of $B^{*}$ even if we could obtain a smooth frame near the considered point. Therefore, we still need deal with the singularities, as we did in our earlier paper.

To make things easier for us, in the rest of this section and in the next section (except for Remark 2), we use notation without ${ }^{*}$ for the maximal direction instead of the minimal direction if there is no confusion.

We let $z_{i}=\nabla_{i} \Psi$. Then

$$
\begin{gathered}
-z_{1}=\nabla_{1}(3 B+A)=\frac{3}{2} \nabla_{1}\left(R_{1 \overline{2} 1 \overline{1}}+R_{2 \overline{1} 2 \overline{1}}-2 R_{1 \overline{1} 1 \overline{1}}\right) \\
\sqrt{-1} \nabla_{1}\left(\operatorname{Im} R_{1 \overline{2} 1 \overline{2}}\right)=\frac{1}{2} \nabla_{1}\left(R_{1 \overline{2} 1 \overline{2}}-R_{2 \overline{1} 2 \overline{1}}\right) \\
=-\frac{1}{3} z_{1}-\nabla_{1} R_{2 \overline{1} 2 \overline{1}}+\nabla_{1} R_{1 \overline{1} 1 \overline{1}} \\
=-\frac{1}{3} z_{1}-\nabla_{2} \bar{R}_{1 \overline{1} 1 \overline{2}}-\nabla_{2} R_{1 \overline{1} 1 \overline{2}} \\
=-\frac{1}{3} z_{1}+(A+B) y_{22}+(B+A) y_{21} \\
-z_{2}=\nabla_{2}(3 B+A)=\frac{3}{2} \nabla_{2}\left(R_{2 \overline{1}} \overline{1}+R_{1 \overline{1} 1 \overline{2}}-2 R_{1 \overline{1} 1 \overline{1}}\right) \\
\sqrt{-1} \nabla_{2}\left(\operatorname{Im} R_{1 \overline{2} 1 \overline{2}}\right)=\frac{1}{2} \nabla_{2}\left(R_{1 \overline{2} 1 \overline{2}}-R_{2 \overline{1} 2 \overline{1}}\right) \\
=\frac{1}{3} z_{2}-\nabla_{2} R_{1 \overline{1} 1 \overline{1}}+\nabla_{2} R_{1 \overline{2}} 1 \overline{2} \\
=\frac{1}{3} z_{2}-\nabla_{1} R_{2 \overline{1} 1 \overline{1}}-\nabla_{1} R_{1 \overline{1} 1 \overline{2}} \\
=\frac{1}{3} z_{2}+(B+A) y_{12}+(A+B) y_{11}
\end{gathered}
$$

We can write the formula in Lemma 2 as

$$
\begin{aligned}
\Delta \Psi & =3\left[\Psi R_{1 \overline{1} 2 \overline{2}}+B(B+3 A)\right] \\
& +3 \frac{A+B}{B} \Psi \sum\left|y_{i 1}+y_{i 2}\right|^{2} \\
- & 2 \frac{A+B}{B} \operatorname{Re}\left[\left(y_{12}+y_{11}\right) \bar{z}_{2}-\left(y_{22}+y_{21}\right) \bar{z}_{1}\right]-\sum \frac{1}{3 B}|z|^{2}
\end{aligned}
$$

Similarly to what we have in the last section, we have two general terms: the first is negative as the constant term of $z$ and $y$; the second is a Hermitian form on $z$ and $y$. We can let $w_{i}=y_{i^{*} 1}-y_{i^{*} 2}$ with $i^{*} \neq i$. Then the second term is a sum of two Hermitian forms. One of these is on $w_{1}, z_{1}$, and the other is on $w_{2}, z_{2}$. We notice that the second term is also nonpositive on $y$ (or nonpositive on $w$, if we assume that $z=0$ ). We can modify the coefficient of $|z|^{2}$ (only) by taking the power of $\Psi$. More precisely, if we let $g=\Psi^{a}$, to make sure that $\Delta g<0$, after taking out a factor $3 \frac{A+B}{B}$, we need

$$
\left|\begin{array}{cc}
\Psi & 1 / 3 \\
1 / 3 & -\frac{1+3 \Psi^{-1}(1-a) B}{9(A+B)}
\end{array}\right| \geq 0
$$

That is,

$$
A+3 B-3(1-a) B-A-B=(3 a-1) B \leq 0
$$

We have $1-3 a \geq 0$. Thus, $a \leq 1 / 3$.

Therefore, we have the following:

Lemma 3. $\Delta g<0$ for $a \leq 1 / 3$ on $M-N$. 
This is exactly the same as what was obtained in [5]. In fact, the number $1 / 6$ was already used in [1-4] for those quadratic functions.

Thus, finally we have the following:

Theorem 1. If $K_{a v}-K_{\min } \leq \frac{1}{2}\left[K_{\max }-K_{\min }\right]$, then $M$ has a constant holomorphic sectional curvature.

Remark 2. The reason we did not come to this earlier was that there was a difficulty when $A=B$. In that case, the argument in [1] ( $p .475$, case 2) seems not to work. Polombo resolved the problem by using a function that is symmetric to $\lambda_{1}=-\frac{A}{3}$ and $\lambda_{2}=\frac{A-3 B}{6}$ (see [2], p. 418, first paragraph and the end of $p$. 397). However, Hong-Cang Yang's function $\Psi$ is only $-6 \lambda_{2}$ and therefore is not symmetric after all. To overcome the difficulty, we let $\Omega=\left\{\left.x \in M\right|_{A=B}\right\}$. Then according to [1], all our calculations are sound on $M-\Omega$, because $N \subset \Omega$. In [5] (p. 2632), there was a suggestion to prove that codim $\Omega \leq 2$, although it was not very well explained. Then, everything went through. The relation was that if we use the argument in [1] $(p .475$, case 2$)$, using the maximal instead of the minimal, we let $B_{1}=\left|R_{1 \overline{2} 1 \overline{2}}\right|$ (or $B^{*}$ as we did earlier); then $2 B_{1}=A-B$. That is, $\Omega=\left\{\left.x \in M\right|_{B_{1}=0}\right\}$. The argument goes as follows:

Case 1: If $\Omega$ is a closed region, we have

$$
\begin{aligned}
0 & \geq \int_{M-\Omega} \Delta g \\
& =a \int_{-\partial \Omega} \Psi^{a-1} \frac{\partial\left(-A_{1}-3 B_{1}\right)}{\partial n} \\
& \geq a \int_{-\partial \Omega}(2 A)^{a-1} \frac{\partial\left(-A_{1}\right)}{\partial n} \\
& =-\int_{\Omega} \Delta F_{1} \geq 0
\end{aligned}
$$

where $F_{1}$ can be chosen from one of the functions in [2] that satisfies the symmetric condition on $M$, for example, a power of $\Phi_{2}$ as in the proof of Proposition 1, or one of our functions with a calculation using the new smooth coordinate in [1] ( $p$. 475) with $R_{1 \overline{1} 1 \overline{1}}=K_{\max }$ (see Equation (4) in the next section). In fact, $A_{1}$ itself is proportional to $\lambda_{2}$ in [2] and is symmetric in the sense of Polombo. On $\Omega, F_{1}$ is just our $g$, as $B_{1}=0$. We notice that there is a sign difference for the Laplace operator in [2]. Again, on $\Omega$, because $A=B$ on a neighborhood, the set of minimal directions is a $S^{1}$ bundle over $\Omega$; therefore, one might choose a smooth section of it locally such that the calculation of [1] still works in our case. That is, one could simply choose $F_{1}$ to be $g$.

Case 2: If $\Omega$ is a hypersurface, the same argument goes through, except that $\int_{\partial(M-\Omega)}(A)^{a-1} \frac{\partial A}{\partial n}=0$, because $A \neq 0$ outside a codimension 1 subset, and on $\Omega_{1}=\left\{\left.x \in \Omega\right|_{A \neq 0}\right\}$, the integral is integrated from both sides.

Therefore, $\Omega$ is a subset of codimension 2, and we can apply Remark 1 . By the calculation in Remark 1, we see that $g$ is harmonic on $M-\Omega$. Now, by Lemma 2, this implies that $B(B-3 A)=0$, and hence $A=B=0$ by our assumptions.

\section{The Generalization}

In fact, in the first section of [1], the authors did not require any negativity. We also see that in our second section, we also do not need any negativity, except when we apply the formula in Lemma 2 in Section 3.

In the first section of [1], they also considered the coordinate in which $R_{1 \overline{1}} 1 \overline{1}$ achieves the maximal instead of the minimal. By using the maximal direction, it is much easier to see that the constant term in the Laplacian is negative. We only need to check the following: 


$$
\begin{aligned}
C & =R_{1 \overline{1} 2 \overline{2}} \\
& =k-R_{1 \overline{1} 1 \overline{1}} \\
& =k / 2-\left(K_{\max }-k / 2\right) \\
& =k / 2-\left(K_{\max }-K_{\min }\right) \\
& =k / 2+A / 3 \leq 0
\end{aligned}
$$

One might compare this with [6] to see the advantage of this new method.

Now, with $C \leq 0$, we could also easily cover the arguments both at the end of the proof of Proposition 1 and in Remark 2 in the case of $B=0$ (using the maximal direction). Similarly to the calculation in Section 2, we obtain the following (see also [15] (p. 27) for a good calculation of this Laplacian at a maximal direction for any complex dimension):

$$
\Delta R_{1 \overline{1} 1 \overline{1}}=-A C+B^{2}=-A C \leq 0
$$

We also have

$$
\begin{gathered}
\nabla R_{1 \overline{1} 1 \overline{2}}=-A \nabla a_{2}-B \nabla \bar{a}_{2}=-A \nabla a_{2} \\
\Delta S_{1 \overline{1} 1 \overline{1}}=-2 A \sum|y|^{2}-A C \\
\nabla_{1} A=-3 \nabla S_{1 \overline{1} 1 \overline{1}}=-3 A y_{21}, \\
\nabla_{2} A=3 A y_{12} \\
\nabla_{\overline{1}} R_{1 \overline{1} 1 \overline{2}}=-A \bar{y}_{22}=0 \\
\nabla_{2} R_{1 \overline{1} 1 \overline{2}}=A y_{11}=0 \\
\Delta\left(|A|^{a}\right)=3 a|A|^{a-1} \Delta S_{1 \overline{1} 1 \overline{1}}+a(a-1)|A|^{a-2}|\nabla A|^{2} \\
=3 a \times(-A)^{a-1}\left(-2 A \sum|y|^{2}-A C\right) \\
+\quad 9 a(a-1)(-A)^{a} \sum|y|^{2} \\
=3 a(-A)^{a}\left[(2-3(a-1)) \sum|y|^{2}+C\right]
\end{gathered}
$$

This is nonpositive when $a \leq 1 / 3$. This is the same as in Lemma 3 and for that in [5].

Therefore, we conclude the general case. One might conjecture that our theorem is also true in the higher-dimensional cases.

Remark 3. We note that this generalization essentially covers the results in [2,4] for the Kähler-Einstein case (see [2], p. 398, Corollary; see also [16], p. 415, Proposition 2 for $W^{+}$for a Kähler surface). One might ask whether our result could be generalized to the Riemannian manifolds with closed half Weyl curvature tensors. This is out of the scope of this paper, although a similar result is true, that is, if $\lambda_{2} \leq 0$ at every point. To make the relation between this paper and [2,4] clearer to the readers, we mention that any one of the half Weyl tensors is harmonic if and only if it is closed, because the tensor is dual to either itself or the negative of itself. Remark (i) in [2] (p. 397) states that if $M$ is Riemannian-Einsteinian, the second Bianchi identity states that the half Weyl tensors are closed (see also [16], p. 408, Equation (9) and p. 411, Remark 1).

\section{Appendix}

Here, we repeat the argument in the proof of Proposition 1 in [6] by using a different but similar argument.

Throughout this Appendix, as in $[1,5,6]$, we assume that $\left\{e_{1}, e_{2}\right\}$ is a unitary basis at a given point $P$ with

$$
R_{1 \overline{1} 1 \overline{1}}=R_{2 \overline{2} 2 \overline{2}}=K_{\min }
$$


or $K_{\max }$.

$$
\begin{gathered}
R_{1 \overline{1} 1 \overline{2}}=R_{2 \overline{2} 2 \overline{1}}=0 \\
A=2 R_{1 \overline{1} 2 \overline{2}}-R_{1 \overline{1} 1 \overline{1}} \geq 0
\end{gathered}
$$

or $\leq 0$ in the maximal direction case.

$$
B=\left|R_{1 \overline{1}} \overline{2}\right|
$$

As in [1], we always have that $A \geq|B|$ or $-A \geq|B|$. We assume that $B \geq 0$.

If $P$ is not a ball-like point, we write

$$
\begin{gathered}
\alpha=e_{1}=\sum a_{i} \partial_{i}, \beta=e_{2}=\sum b_{i} \partial_{i} \\
S_{1 \overline{1} 1 \overline{1}}=R\left(e_{1}, \bar{e}_{1}, e_{1}, \bar{e}_{1}\right)=\sum R_{i j k l} a_{i} \bar{a}_{j} a_{k} \bar{a}_{l}
\end{gathered}
$$

and so on.

In particular, we have

$$
S_{1 \overline{1} 1 \overline{1}}=S_{2 \overline{2} 2 \overline{2}}, S_{1 \overline{1} 1 \overline{2}}=S_{2 \overline{2} 2 \overline{1}}=0
$$

We calculate the Laplace of $\Phi_{1}=\tau^{2}=\frac{|B|^{2}}{A^{2}}$ at a critical point.

We let

$$
x_{i}=\nabla_{i} \Phi_{1}=2 \frac{\tau}{A}\left[\operatorname{Re} \nabla_{i} S_{1 \overline{2} 1 \overline{2}}+3 \tau \nabla_{i} S_{1 \overline{1} 1 \overline{1}}\right]
$$

As in $[1,3,5]$, we have

$$
\begin{aligned}
& \Delta R_{1 \overline{1} 1 \overline{1}}=-A R_{1 \overline{1} 2 \overline{2}}+B^{2} \\
& \Delta R_{1 \overline{1} 1 \overline{2}}=3\left(R_{1 \overline{1} 2 \overline{2}}-A\right) B
\end{aligned}
$$

At $P$, we have $a_{1}=b_{2}=1$ and $a_{2}=b_{1}=0, \nabla a_{1}=\nabla b_{2}=0, \nabla a_{2}+\nabla \bar{b}_{1}=0$. Therefore, we write $y_{i 1}=\nabla_{i} a_{2}$ and $y_{i 2}=\nabla_{i} \bar{a}_{2}$. We also have

$$
\begin{gathered}
\Delta\left(a_{1}+\bar{a}_{1}\right)=-\left|\nabla a_{2}\right|^{2}, \Delta\left(a_{2}+\bar{b}_{2}\right)=0 \\
\nabla_{i} R_{1 \overline{1} 1 \overline{2}}=-A y_{i 1}-B y_{i 2}
\end{gathered}
$$

because

$$
0=\nabla S_{1 \overline{1} 1 \overline{2}}=\nabla R_{1 \overline{1} 1 \overline{2}}+2 R_{2 \overline{1} 1 \overline{2}} \nabla a_{2}+B \nabla \bar{a}_{2}+R_{1 \overline{1} 1 \overline{1}} \nabla \bar{b}_{1}
$$

that is,

$$
\nabla R_{1 \overline{1} 1 \overline{2}}=-A \nabla a_{2}-B \nabla \bar{a}_{2}
$$

This also gives a similar formula for $\nabla_{\bar{i}} R_{1 \overline{1} 1 \overline{2}}$. Similarly,

$$
\begin{gathered}
\nabla S_{1 \overline{1} 1 \overline{1}}=\nabla R_{1 \overline{1} 1 \overline{1}} \\
\nabla S_{1 \overline{2} 1 \overline{2}}=\nabla R_{1 \overline{1} 1 \overline{2}} \\
\Delta S_{1 \overline{1} 1 \overline{1}}=-2 A \sum|y|^{2}-4 B \operatorname{Re} \sum y_{i 1} \bar{y}_{i 2}-A R_{1 \overline{1} 2 \overline{2}}+B^{2} \\
\operatorname{Re} \Delta S_{1 \overline{2} 1 \overline{2}}=4 A \sum \operatorname{Re} y_{i 1} \bar{y}_{i 2}+2 B \sum|y|^{2}+3\left(R_{1 \overline{1} 2 \overline{2}}-A\right) B \\
\nabla_{\overline{1}} S_{1 \overline{1} 1 \overline{2}}=-A \bar{y}_{22}-B \bar{y}_{21} \\
\nabla_{2} S_{1 \overline{2} 1 \overline{2}}=A y_{11}+B y_{12} \\
\nabla_{1} S_{1 \overline{2} 1 \overline{2}}=-A\left(6 \tau^{2}-1\right) y_{22}-5 A \tau y_{21}+x_{1} \\
\nabla_{\overline{2}} S_{1 \overline{1} 1 \overline{2}}=5 A \tau \bar{y}_{12}+A\left(6 \tau^{2}-1\right) \bar{y}_{11}+\bar{x}_{2}
\end{gathered}
$$

As in [3] (p. 598), at $P$ we have 


$$
\begin{aligned}
\Delta \Phi_{1} & =\frac{2 \tau \Delta B}{A}+\frac{6 \tau^{2}}{A} \Delta S_{1 \overline{1} 1 \overline{1}} \\
& +\frac{1}{A^{2}} \sum\left(\left|\nabla S_{1 \overline{2} 1 \overline{1}}\right|^{2}+\left|\bar{\nabla} S_{1 \overline{2} 1 \overline{1}}\right|^{2}\right)+\frac{54 \tau^{2}}{A^{2}} \sum\left|\nabla S_{1 \overline{1} \overline{1}}\right|^{2} \\
& +\frac{12 \tau}{A^{2}} \sum \operatorname{Re}\left(\nabla_{i} S_{1 \overline{1} 1 \overline{1}}\left(\nabla_{\bar{i}}\left(S_{1 \overline{1} 1 \overline{2}}+S_{2 \overline{1} 2 \overline{1}}\right)\right)\right. \\
& =2 \tau\left[3 A \tau\left(\tau^{2}-1\right)-4 \tau \sum|y|^{2}+4\left(1-3 \tau^{2}\right) \sum \operatorname{Re}\left(y_{i 1} \bar{y}_{i 2}\right)\right] \\
& +\left|y_{22}+\tau y_{21}\right|^{2}+\left|y_{11}+\tau y_{12}\right|^{2} \\
& +\frac{1}{A^{2}}\left[\left|x_{1}+A\left[\left(1-6 \tau^{2}\right) y_{22}-5 \tau y_{21}\right]\right|^{2}+\left|x_{2}+A\left[\left(6 \tau^{2}-1\right) y_{11}+5 \tau y_{12}\right]\right|^{2}\right. \\
& -18 \tau^{2}\left[y_{12}+\left.\tau y_{11}\right|^{2}+\left|y_{21}+\tau y_{22}\right|^{2}\right] \\
& +\frac{12 \tau}{A}\left[\operatorname{Re}\left[\left(y_{21}+\tau y_{22}\right) \bar{x}_{1}\right]-\operatorname{Re}\left[\left(y_{21}+\tau y_{11}\right) \bar{x}_{2}\right]\right]
\end{aligned}
$$

Here we notice that $\Delta \Phi_{1}$ has two general terms. The first term has nothing to do with $x$ or $y$ and therefore can be regarded as a constant term to these. This term is always nonpositive, because $\frac{1}{3} \leq \tau \leq 1$.

The second term can be regarded as a Hermitian form $h$ to $x$ and $y$. We can separate $x$ and $y$ into two groups: $x_{1}, y_{2 j}$ in one group and $x_{2}, y_{1 j}$ in the other. These two groups of variables are orthogonal to each other with respect to this Hermitian form. That is, $h=h_{1}+h_{2}$ with $h_{1}$ (or $h_{2}$ ) only depends on the first (second) group of variables.

We need to check the nonpositivity for each of these.

For $x_{2}, y_{11}, y_{12}$, the corresponding matrix of $h_{2}$ is

$$
\left[\begin{array}{ccc}
\frac{1}{A^{2}} & -\frac{1}{A} & -\frac{\tau}{A} \\
-\frac{1}{A} & 2\left(9 \tau^{2}-1\right)\left(\tau^{2}-1\right) & 0 \\
-\frac{\tau}{A} & 0 & 0
\end{array}\right]
$$

The matrix for $h_{1}$ of $x_{1}, y_{21}, y_{22}$ is

$$
\left[\begin{array}{ccc}
\frac{1}{A^{2}} & \frac{\tau}{A} & \frac{1}{A} \\
\frac{\tau}{A} & 0 & 0 \\
\frac{1}{A} & 0 & 2\left(9 \tau^{2}-1\right)\left(\tau^{2}-1\right)
\end{array}\right]
$$

When $P$ is a critical point of $\Phi_{1}$, then $x_{1}=x_{2}=0$. The matrices on $y$ are clearly semidefinite.

Acknowledgments: I thank Professors Poon and Wong and the Department of Mathematics, University of California at Riverside for their support. I thank Professor Hong-Cang Yang for showing me his work when I was a graduate student in Berkeley. I also thank Professor Paul Yang for sharing with me the work [4]. I also thank Tommy Murphy for discussions and the referees of [6] and this paper for their useful comments. Finally, this paper was written up when I visited Xiamen University. Here I take this chance to thank Professor Qiu Chunhui and the department for their warm hospitality.

Conflicts of Interest: The author declares no conflict of interest.

\section{References}

1. Siu, Y.T.; Yang, P. Compact Kähler-Einstein surfaces of nonpositive bisectional curvature. Invent. Math. 1981, 64, 471-487.

2. Polombo, A. De Nouvelles Formules De Weitzenböck Pour Des Endomorphismes Harmoniques Applications Géométriques. Ann. Sci. Ec. Norm. 1992, 25, 393-428.

3. Hong, Y.; Guan, Z.D.; Yang, H.C. A note on the Kähler-Einstein Manifolds. Acta. Math. Sin. 1988, 31, 595-602.

4. Polombo, A. Condition d'Einstein et courbure negative en dimension 4. C. R. Acad. Sci. Paris Ser. 1 Math. 1988, 307, 667-670. 
5. Chen, D.; Hong, Y.; Yang, H.C. Kähler-Einstein surface and symmetric space. Sci. China Math. 2011, 54, 2627-2634.

6. Guan, D. On Bisectional Negatively Curved Compact Kähler-Einstein Surfaces. Pac. J. Math. 2017, 288, 343-353.

7. Dancer, A.; Wang, M. Kähler Einstein Metrics of Cohomogeneity One and Bundle Construction for Einstein Hermitian Metrics. Math. Ann. 1998, 312, 503-526.

8. Apostolov, V.; Calderbank, D.; Gauduchon, P.C. Tonnesen-Friedman: Hamilton 2-forms in Kähler geometry III, extremal metrics and stability. Invent. Math. 2008, 173, 547-601.

9. Bogomolov, F. On Guan's examples of simply connected non-kähler compact complex manifolds. Am. J. Math. 1996, 118, 1037-1046.

10. Console, S.; Fino, A. On the de Rham cohomology of solvmanifolds. Ann. Scuola Norm. Super. Pisa 2011, 10, 801-818.

11. Guan, D. A Note On the classification of Compact Complex Homogeneous Locally Conformal Kähler Manifolds. J. Math. Stat. 2017, 13, 261-267.

12. Huckleberry, A. Homogeneous Pseudo-Kähler Manifolds: A Hamiltonian Viewpoint. Note Math. 1990, 10 (Suppl. 2), 337-342.

13. Podesta, F.; Spiro, A. Kähler manifolds with large isometry group. Osaka J. Math. 1999, 36, 805-833.

14. Tian, G. Smoothness of the Universal Deformations Space of Compact Calabi-Yau Manifolds and Its Peterson-Well Metric. In Mathematical Aspect of String Theory; Yau, S.T., ed.; World Scientific: Singapore, 1987; pp. 629-646.

15. Mok, N.; Zhong, J.Q. Curvature Characterization of Compact Hermitian Symmetric Spaces. J. Diff. Geom. 1986, 23, 15-67.

16. Derdzinski, A. Self-dual Kähler manifolds and Einstein manifolds of dimension four. Compos. Math. 1983, 49, 405-433.

(C) 2018 by the authors. Licensee MDPI, Basel, Switzerland. This article is an open access article distributed under the terms and conditions of the Creative Commons Attribution (CC BY) license (http:/ / creativecommons.org/licenses/by/4.0/). 